\title{
INTERPOLATION ON FINITE OPEN RIEMANN SURFACES
}

\author{
E. L. STOUT ${ }^{1}$
}

I. In a previous paper [5], we have considered the question of interpolation on finite open Riemann surfaces; it is our intention to show in this note that the problem can be treated in an essentially simpler way.

We shall suppose given a finite open Riemann surface, $R$. Such a surface is, by definition, the complement in some compact Riemann surface of a finite family of closed, pairwise disjoint discs each of which we may assume to have an analytic simple closed curve as boundary. A set $E \subset R$ is called an interpolation set for $R$ if given a bounded, complex valued function $\alpha$ on $E$, there is a bounded holomorphic function $f$ on $R$ such that $f \mid E=\alpha$. It is convenient to denote by $H_{\infty}[R]$ the collection of all functions which are bounded and holomorphic on $R$.

For our characterization of interpolation sets in $R$ we introduce a functional $d_{R}(z, E)$ as follows: If $z \in E$, then

$$
d_{R}(z, E)=\sup \left\{|f(z)|: f \in H_{\infty}[R], f \mid(E-\{z\})=0,\|f\|_{R} \leqq 1\right\}
$$

where $\|f\|_{R}=\sup \{|f(w)|: w \in R\}$ is the usual supremum norm.

II. We can characterize the interpolation sets for $R$ in terms of the functional $d_{R}$ as follows.

ThEOREM. The set $E \subset R$ is an interpolation set for $R$ if and only if $\inf \left\{d_{R}(z, E): z \in E\right\}>0$.

Proof. Denote by $\Gamma$ the boundary of $R$ so that $\Gamma$ consists of a finite collection of pairwise disjoint analytic simple closed curves. It is convenient to make use of an Ahlfors map for $R$, i.e., a function $\pi$ continuous on $\bar{R}$ and holomorphic in $R$ which is constantly of modulus one on $\Gamma$. The existence of such functions was established by Ahlfors in [1]; an alternative proof of their existence is in [4]. It is easy to see that $d \pi$, the differential of $\pi$, does not vanish on $\Gamma$, and thus $\pi$ acts as a local homeomorphism at every point of $\Gamma$.

Let us suppose, initially, that $E$ satisfies the following very stringent condition: We assume the existence of discs $V \subset \bar{V} \subset W$ in the ambient compact surface both of which meet $\Gamma$ in open arcs and

Received by the editors March 1, 1966.

1 This research was supported by National Science Foundation Grant NSF-GP5493. 
which, moreover, have the properties that $E \subset V$ and $\pi$ is one-to-one on $\bar{W}$. The hypothesis of the theorem implies that for each $z \in E$, $d_{W \cap R}(z, E)>\delta$ for some fixed $\delta>0$ and all $z \in E$. Consequently, by a well-known theorem of Carleson [3, p. 203], the set $E$ is an interpolation set for $W \cap R$. (For this formulation of Carleson's theorem, see $[5$, Lemma 5.1].) It follows then that the set $\pi[E]$ is an interpolation set for $\pi[W \cap R]$. Since $\pi[E] \subset \pi[V]$, and since $\pi$ is one-to-one on $\bar{W}$, it follows from [5, Theorem 5.3] that $\pi[E]$ is an interpolation set for $U$, the open unit disc. But this clearly implies that $E$ is an interpolation set for $R$.

We now show how this very special case of our theorem implies the general case. For each $p \in \Gamma$ there exist discs $V_{p} \subset \bar{V}_{p} \subset W_{p}$ as above such that $E \cap V_{p}$ is an interpolation set for $R$. Finitely many of the neighborhoods $V_{p}$, denote them by $V_{1}, \cdots, V_{m}$, cover $\Gamma$. Since the assumption of the theorem clearly implies that the set $E$ is discrete, it follows that all but finitely many points of $E$ lie in $\bigcup_{j=1}^{m} V_{j}$. The union of an interpolation set and a finite set is again an interpolation set whence it suffices for us to prove $E$ to be an interpolation set for $R$ under the assumption that $E \subset \bigcup_{j=1}^{m} V_{j}$. Let us write $E=E_{1} \cup \ldots$ $\cup E_{m}$ where this is a disjoint union and where $E_{j} \subset V_{j}$.

We shall prove the existence of functions $b_{1}, \cdots, b_{m} \in H_{\infty}[R]$ with the properties that $\left|b_{j}\right|>\epsilon$ on $E_{j}$ and $b_{j}$ vanishes on $E_{k}$ if $k \neq j$. Here $\epsilon$ is some fixed positive number independent of $j$. Let us suppose the functions $b_{j}$ have been constructed, and consider a bounded function $\alpha$ on $E$. Since $E_{j}$ is an interpolation set for $R$ and since $b_{j}$ is bounded away from 0 on $E_{j}$, there exists $h_{j} \in H_{\infty}[R]$ such that $h_{j}(z)$ $=\alpha(z) / b_{j}(z)$ for all $z \in E_{j}$. If $H=\sum_{j=1}^{m} b_{j} h_{j}$, then $H$ is a bounded analytic function on $R$ which agrees on $E$ with $\alpha$.

We conclude the proof of the sufficiency of our condition by establishing the existence of the functions $b_{j}$. In this proof we use the uniformization theorem. The universal covering surface of $R$ is the open unit disc, $U$. Let $\Upsilon: U \rightarrow R$ be a uniformizing map, and let $(S)$ be the associated group of covering transformations. Denote by $D$ the canonical fundamental domain for the group (5) so that $D$ is a certain open set together with a portion of its boundary which is mapped by $\Upsilon$ on to $R$ in a one-to-one fashion. Denote by $E_{j}^{\prime}$ the set $\Upsilon^{-1}\left[E_{j}\right] \cap D$ and by $E_{j}^{\prime \prime}$ the set $\Upsilon^{-1}\left[E_{j}\right]$.

Let $B_{j}$ be the Blaschke product with zero set, the set $\bigcup\left\{E_{\mathbf{k}}^{\prime \prime}: k \neq j\right\}$ and with simple zeros. It is the case that if $\delta$ is a lower bound for the numbers $d_{R}(z, E)$ for $z$ in $E$, then $\left|B_{j}(w)\right|>\delta$ for all $w$ in $E_{j}^{\prime \prime}$. To establish this assertion, let $z \in E_{j}^{\prime \prime}$ be given. There is $f \in H_{\infty}[R]$ which is bounded by one, which is at least $\delta$ in modulus at the point $\Upsilon(z)$, 
and which vanishes on the set $E-\{\Upsilon(z)\}$. Thus, the element $f \circ \Upsilon$ of $H_{\infty}[U]$ vanishes on $U\left\{E_{\boldsymbol{k}}^{\prime \prime}: k \neq j\right\}$, is at least $\delta$ in modulus at $z$, and is bounded by one. We can write $f \circ \Upsilon=B S F$ where $B$ is a Blaschke product, $S$ is a singular function, and $F$ is an outer function. Since $\|f \circ \Upsilon\|_{U} \leqq 1$, it follows that $\|F\|_{U} \leqq 1$. Also, $S$ is bounded by one. It follows therefore that $|B(z)|$ is at least $\delta$. The zero set of $B$ contains that of $B_{j}$, and since $B_{j}$ has only simple zeros, we may write $B=B_{j} B^{\prime}$ where $B^{\prime}$ is some other Blaschke product. But this obviously implies that $\left|B_{j}(z)\right|$ is at least $\delta$ in modulus. This calculation is independent of the choice of $z$ in $E_{j}^{\prime \prime}$, so the assertion concerning $B_{j}$ is established.

The function $B_{j}$ is not invariant under the group (5). It does, however, have the weaker property that $\left|B_{j}\right|$ is invariant. This is a consequence of the fact that if $[z, w]=|(z-w) /(1-z \bar{\omega})|$ and if $g$ is a conformal mapping of the open unit disc on to itself, then $[g(z), g(w)]$ $=[z, w]$. For the details, see $[6$, p. 510]. It is easily verified that the map $\chi$ from (f) to the unit circle given by $\chi(g)=B_{j}(g(z)) / B_{j}(z)$ is independent of the choice of $z \in U$; moreover this map is a character of (3): $\chi\left(g_{1} g_{2}\right)=\chi\left(g_{1}\right) \chi\left(g_{2}\right)$. In the terminology of [2], the function $B_{j}$ is automorphic with character $\chi$, i.e., $B_{j}(g(z))=\chi(g) B_{j}(z)$ for all $g \in(B)$ and all $z \in U$. If we now invoke Lemma 5 of [2], there is a unit $C_{j}$ of $H_{\infty}[U]$ which is automorphic with character $\bar{\chi}: C_{j}(g(z))=\bar{\chi}(g) C_{j}(z)$. Then $\left|C_{j}\right|>\delta^{\prime}$ on the set $E_{j}^{\prime \prime}$ for some $\delta^{\prime}>0$. The function $b_{j}^{\prime}$ defined by $b_{j}^{\prime}(z)=C_{j}(z) B_{j}(z)$ is easily verified to be invariant under the group (3). Thus if we define $b_{j}$ on $R$ by $b_{j}=b_{j}^{\prime} \circ \Upsilon^{-1}, b_{j}$ is a well-defined element of $H_{\infty}[R]$ which vanishes on the sets $E_{k}$ for $k \neq j$ and whose modulus is at least $\delta \delta^{\prime}$ on the set $E_{j}$. Thus, we have established the sufficiency of our condition.

The necessity follows from general Banach space considerations; one may consult $[3$, p. 196] for the necessary argument.

III. From the theorem of the preceding section, we shall derive a second characterization of interpolation sets in $R$. We have that $\Gamma$, the boundary of $R$, consists of a finite collection $\left\{\gamma_{1}, \cdots, \gamma_{m}\right\}$ of pairwise disjoint analytic simple closed curves. For $j=1, \cdots, m$, let $\gamma_{j}^{\prime}$ be a simple closed curve in $R$ which, together with $\gamma_{j}$, bounds an annular region $A_{i}$ in $R$. We suppose the $A_{j}$ to have disjoint closures.

THEOREM. The discrete set $E \subset R$ is an interpolation set for $R$ if and only if $E_{j}=A_{j} \cap E$ is an interpolation set for $A_{j}$ for each $j$.

Proof. If $E$ is an interpolation set for $R$, then, a fortiori, $E_{j}$ is one for $A_{j}$. 
To establish the opposite implication, we shall show that if each $E_{j}$ is an interpolation set for $A_{j}$, then for some fixed $\delta>0$ and for all $z \in E, d_{R}(z, E)>\delta$ so that the previous result may be invoked to yield the result.

By hypothesis, $R$ is obtained from a compact surface $R_{0}$ by deleting closed discs $V_{1}, \cdots, V_{m}$ where, say, the boundary of $V_{j}$ is the curve $\gamma_{j}$. Let $R_{j}=R \cup V_{1} \cup \ldots \cup V_{j-1} \cup V_{j+1} \cup \ldots \cup V_{m}$ so that $R_{j}$ is a finite open Riemann surface which contains $R$. Let $\pi_{1}: R_{1} \rightarrow U$ be an Ahlfors map for the surface $R_{1}$. Since $\pi_{1}$ acts as a local homeomorphism in a neighborhood of each point of $\gamma_{1}$, it follows that for some annulus $A_{1}^{\prime}$ contained in $A_{1}$ and having $\gamma_{1}$ as one of its bounding components, we may write $A_{1}^{\prime}=Q_{1} \cup \ldots \cup Q_{M}$ where for later notational convenience we take our indices to be integers $\bmod M$ and where

(i) each $Q_{j}$ is a closed curvilinear quadrilateral the boundary of which meets $\gamma_{1}$ in an arc,

(ii) $Q_{j}$ and $Q_{j+1}$ intersect in an arc connecting the components of the boundary of $A_{j}^{\prime}$, and

(iii) $\pi_{1}$ is one-to-one on $U\left\{Q_{j}: j=j_{0}, \cdots, j_{0}+5\right\}$ for each $j_{0}$.

Since $E_{1}$ differs from $E \cap A_{1}^{\prime}$ by at most a finite set, $E_{1}$ is an interpolation set for $R_{1}$ if and only if $E \cap A_{1}^{\prime}$ is. We shall show $E \cap A_{1}^{\prime}$ to be an interpolation set for $R_{1}$.

The hypotheses on the $Q_{j}$ together with the hypothesis on the set $E$ imply, as in the previous proof, that $E \cap\left(Q_{i+1} \cup Q_{i} \cup Q_{i+1}\right)$ is an interpolation set for the surface $R_{1}$. Consequently, there is $\beta_{1}>0$ such that for each $i=1, \cdots, M$, and for each $z \in E \cap Q_{i}$, there is $f_{i, z}$ $\in H_{\infty}\left[R_{1}\right]$ which is bounded by one, which is at least $\beta_{1}$ in modulus at $z$ and which vanishes at all points of the set $E \cap\left(Q_{i-1} \cup Q_{i} \cup Q_{i+1}\right)$ other than $z$.

Next, we assert the existence of functions $g_{1}, \cdots, g_{M} \in H_{\infty}\left[R_{1}\right]$ such that for some $\alpha_{1}>0,\left|g_{j}(z)\right|>\alpha_{1}$ if $z \in E \cap Q_{j}$ but $g_{j}(z)=0$ if $z \in E \cap Q_{k}$ for $k \neq j-1, j, j+1$. Suppose the desired $g_{j}$ exist. It is clearly no loss of generality to suppose them bounded by one on $R_{\mathbf{1}}$. If $z \in E \cap A_{1}^{\prime}$, then $z \in Q_{j}$ for some $j$. We have $\left|g_{j}(z) f_{j, z}(z)\right|>\alpha_{1} \beta_{1}$, $\left\|g_{j} f_{j, z}\right\|<1$, and that $g_{j} f_{j, z}$ vanishes on the set $\left(E \cap A_{j}^{\prime}\right)-\{z\}$. Thus the set $E \cap A_{j}^{\prime}$ is an interpolation set for $R_{1}$.

To prove the existence of the desired functions $g_{j}$, we argue as follows. Let $p$ and $q$ be interior points of the arcs $Q_{j-1} \cap \partial R_{1}$ and $Q_{j+1} \cap \partial R_{1}$ respectively. Let $\lambda$ be the arc of $\partial R_{1}$ containing $Q_{j} \cap \partial R_{1}$ and having $p$ and $q$ as end points. Let $\lambda^{\prime}$ be an arc in $R_{0}-\bar{R}_{1}$ with the same end points. (Recall that $R_{0}$ is the originally given compact surface.) The $\operatorname{arcs} \lambda$ and $\lambda^{\prime}$ jointly constitute the boundary of a topological disc $D$. The surface $R_{1}^{\prime}$ obtained by forming the union of $R_{1}$, of $D$, and of the 
interior of $\lambda$ is again a finite open Riemann surface. It contains $Q_{j}$ as a compact subset. Using an Ahlfors map for $R_{1}^{\prime}$, we find that the set $F_{j}$ $=E \cap\left(Q_{1} \cup \ldots \cup Q_{j-2} \cup Q_{j+2} \cup \ldots \cup Q_{M}\right)$ is a certain finite union of interpolation sets for $R_{1}^{\prime}$. Thus, this set is surely contained in the zero set of some nonzero element of $H_{\infty}\left[R_{1}^{\prime}\right]$. The fact that $Q_{j}$ is compactly contained in $R_{1}^{\prime}$ now implies the existence of a function in $H_{\infty}\left[R_{1}^{\prime}\right]$ which is zero free on $Q_{j}$ and which vanishes on the set $F_{j}$. The restriction of this function to $R_{1}$ will serve as the function $g$, which we seek.

Thus, $E \cap A_{1}^{\prime}$ is an interpolation set for $R_{1}$ and so $E \cap A_{1}$ is also one. Similar remarks hold for the sets $E \cap A_{j}$ and the surfaces $R_{j}$. It follows that there exist functions $h_{j} \in H_{\infty}\left[R_{j}\right]$ which are bounded by one, which vanish on $E \cap A_{j}$ and which, for some $\delta>0$ independent of $j$, satisfy $\left|h_{j}\right|>\delta$ on $E \cap A_{k}$ for $k \neq j$. By making $\delta$ sufficiently small we can also assert the existence, for each $z \in E \cap A_{j}$ of a function $h_{j, z}$ bounded by one on $R$ which is at least $\delta$ in modulus at $z$ and which vanishes at every other point of $E \cap A_{j}$. If $z \in E \cap A_{j}$, then the function $h_{1} \cdots h_{j-1} h_{j, z} h_{j+1} \cdots h_{m}$ is bounded by one, is at least $\delta^{m}$ in modulus at $z$, and vanishes on the set $\left(E \cap\left(\cup A_{j}\right)\right)-\{z\}$. This estimate is uniform in $z$, so we may conclude from the previous theorem that $E \cap\left(U A_{j}\right)$ is an interpolation set for $R$. Consequently, $E$ itself is an interpolation set for $R$.

IV. As noted at the beginning, these results are not new; it is the proofs which are our concern here. In the original development [5], our first theorem was obtained as an immediate corollary of our second, but the proof of the second was much more complicated than the present arguments. Several other characterizations of interpolation sets can be derived from these results as in [5].

\section{REFERENCES}

1. L. V. Ahlfors, Open Riemann surfaces and extremal problems on compact subregions, Comment. Math. Helv. 24 (1950), 100-134.

2. F. Forelli, Another proof of the corona conjecture for finite open Riemann surfaces, Illinois J. Math. 10 (1966), 367-380.

3. K. Hoffman, Banach spaces of analytic functions, Prentice-Hall, Englewood Cliffs, N. J., 1962.

4. H. L. Royden, The boundary values of analytic and harmonic functions, Math. $Z$. 78 (1962), 1-24.

5. E. L. Stout, Bounded holomorphic functions on finite Riemann surfaces, Trans. Amer. Math. Soc. 120 (1965), 255-285.

6. M. Tsuji, Potential theory in modern function theory, Maruzen, Tokyo, 1959.

YALE UNIVERSITY 